\title{
Measurement of Large-Signal Coss and Coss Losses of Transistors Based on Nonlinear Resonance
}

\author{
Mohammad Samizadeh Nikoo, Armin Jafari, Nirmana Perera, Member, IEEE, and Elison Matioli, Member, IEEE
}

\begin{abstract}
In this letter, we present a new measurement technique to evaluate the large-signal output capacitance ( $\left.C_{\mathrm{OSs}}\right)$ of transistors as well as the $C$ oss energy dissipation ( $\left.E_{\mathrm{DISS}}\right)$, based on the nonlinear resonance between a known inductor and the output capacitance of the device under test. The method is simple and robust, and only requires a single voltage measurement to extract the large-signal $C$ oss both in charging and discharging transients. By changing the circuit parameters, it is possible to tune the resonance frequency (even above $40 \mathrm{MHz}$ ) and the voltage swing (even above $1 \mathrm{kV}$ ) with $\mathrm{d} v / \mathrm{d} t$ exceeding $100 \mathrm{~V} / \mathrm{ns}$, even though the method relies only on a low-voltage DC source, without the need for high-voltage RF amplifiers. The single-pulse operation of the method enables measuring $\boldsymbol{C}_{\mathrm{OSs}}$ and $\boldsymbol{E}_{\mathrm{DISs}}$ at very high frequency and $\mathrm{d} v / \mathrm{d} t$ values without any thermal runaway. Using the proposed method, we extracted large-signal $C_{\text {oss }}$ and $E_{\text {Diss of }}$ power transistors based on different semiconductor technologies. The obtained results were verified by Sawyer-Tower method and data reported in the literature. The precise characterization of large-signal $C_{\text {oss }}$ of transistors presented in this letter is essential for the design of power converters, especially those operating at high switching frequencies.
\end{abstract}

Index Terms-Output capacitance (Coss), Coss losses, Energy dissipation ( $E_{\text {DISs }}$ ), Large-signal, Hysteresis, Nonlinear resonance, $\mathrm{GaN}, \mathrm{Si}$, SiC, Superjunction, HEMT, Sawyer Tower.

\section{INTRODUCTION}

$\mathrm{L}$ ARGE-SIGNAL analysis of the output capacitance $\left(C_{\mathrm{OSS}}\right)$

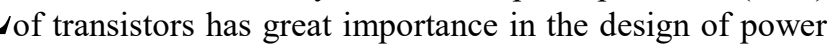
converters, as the small-signal $C_{\text {OSs }}$ values are not always valid in the switching transients, especially when hysteresis exists [1]-[4]. The Coss hysteresis can lead to a considerable power loss which is not recoverable with soft switching topologies [5]-[8]. The significant energy dissipation observed in the charging and discharging process of the output capacitance of some Si superjunction (SJ) MOSFETs initiated studies on their large-signal $C_{\text {oss }}$ [8], [9]. The lower-thanexpected efficiency in soft-switched GaN-based power converters, again highlight the need for precise measurements of $C_{\text {Oss }}$ losses [1], [2].

Sawyer-Tower (ST) is a typical electrical measurement method used to evaluate large-signal output capacitance of transistors and diodes [10], [11]. In this method, the gate and source of the transistor are shorted to turn OFF the device, and a high-amplitude sinusoidal waveform is applied to the series connection of the device under test (DUT) and a reference linear capacitor $\left(C_{\mathrm{REF}}\right)$. The stored charge in the $C_{\mathrm{OSS}}$ of the transistor is assumed to be equal to the charge stored in the reference capacitor. This assumption is valid when the leakage current in

The authors are with the Power and Wide-band-gap Electronics Research Laboratory (POWERlab), École polytechnique fédérale de Lausanne (EPFL), CH-1015 Lausanne, Switzerland (e-mail: elison.matioli@epfl.ch).
DUT is much smaller than the displacement current, otherwise it may lead to the observation of spurious hysteresis [12]. The choice of $C_{\mathrm{REF}}$ is crucial in the ST method, since it depends on the range of interest for $C_{\mathrm{OSS}}$, which however has a significant nonlinearity leading to a variation by a few orders of magnitude when changing drain-to-source bias. As a result, a fixed value of $C_{\mathrm{REF}}$ is not always suitable to capture such large nonlinearity. Another major limitation of the ST method, is that the measurements are performed in steady-state regime. In case of devices with $C_{\text {oss }}$ hysteresis, the junction can significantly heat up due to hysteresis losses, which could potentially affect the measurement accuracy. For instance, the leakage current is much larger at higher temperatures, and can become comparable to the displacement currents in $C_{\mathrm{OSS}}$ and $C_{\mathrm{REF}}$, resulting in an incorrect $C_{\text {oss }}$ measurement [12]. Another restriction caused by any steady-state measurement is the limitation of voltage-swing/frequency applied to the DUT before thermal runaway [1]. In addition, the need for highamplitude sinusoidal voltage source in the ST method constrains the maximum voltage and frequency that can be applied to characterize large-signal $C_{\mathrm{OSs}}$, and the typical values of $\mathrm{d} v / \mathrm{d} t$ in switching transients, especially for GaN transistors, can only be achieved at very high frequencies, in the range of tens of megahertz [2].

In this letter we present a simple and robust measurement method to capture large-signal $C_{\text {oss }}$ as well as $C_{\text {oss }}$ energy dissipation during a single charging/discharging process ( $\left.E_{\mathrm{DISS}}\right)$. The method works based on the nonlinear resonance between a pre-calibrated inductor and the output capacitance of the DUT. By changing the circuit parameters, it is possible to tune the $\mathrm{d} v / \mathrm{d} t$ (above $100 \mathrm{~V} / \mathrm{ns}$ ), frequency (above $40 \mathrm{MHz}$ ), and the voltage swing (above $1 \mathrm{kV}$ ), even though the method relies only on a low-voltage DC source, without the need for RF power amplifiers. In addition, large-signal $C_{\mathrm{OSS}}$ as well as $E_{\mathrm{DISS}}$ can be measured in steady-state regime as well as under a single pulse condition, which eliminates the effect of self-heating from the measurement, and does not thermally limit the maximum voltage swing, frequency, and $\mathrm{d} v / \mathrm{d} t$ applied to the DUT.

\section{Methodology}

Fig. 1a illustrates the proposed circuit for large-signal $C_{\text {Oss }}$ and $E_{\text {DISS }}$ measurement. The transistor $M$ is ON for $t<t_{0}$, charging the inductor $L$ with the current $I_{0}$. By turning OFF the transistor at $t=t_{0}$, the inductor resonates with $C_{\mathrm{OSS}}$ and the parasitic capacitances $C_{\mathrm{par}}$. This results in the generation of a pulse with an amplitude $V_{\max }$ at the output node. By measuring the drain-source voltage waveform ( $\left.v_{\mathrm{DS}}\right)$, and knowing the values of $L$ and $C_{\mathrm{par}}$, one can simply extract large-signal $C_{\mathrm{OSs}}$ as 

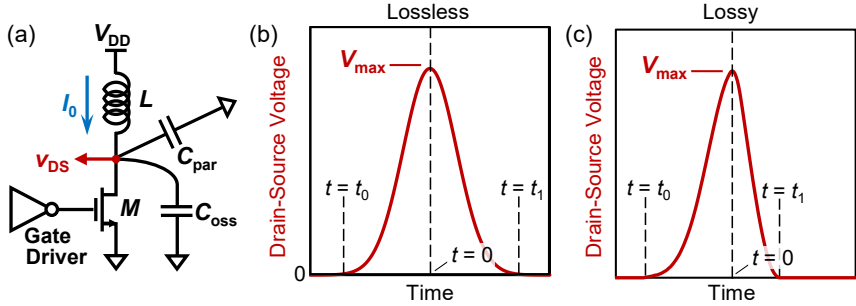

(d)

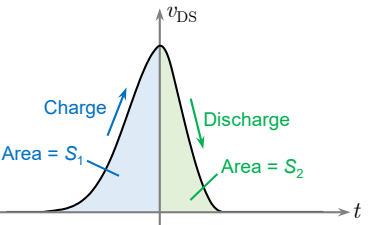

(e)

\begin{tabular}{c|c} 
Energy & Calculation \\
\hline$E_{\mathrm{OSS}}^{\text {ch }}$ & $\frac{1}{2 L} S_{1}^{2}$ \\
\hline$E_{\mathrm{OSS}}^{\text {disc }}$ & $\frac{1}{2 L} S_{2}^{2}$ \\
\hline$E_{\mathrm{DISS}}$ & $\frac{1}{2 L}\left(S_{1}^{2}-S_{2}^{2}\right)$
\end{tabular}

Fig. 1. Proposed method for large-signal $C_{\text {oss }}$ and $E_{\text {DISs }}$ measurement. (a) The circuit, and samples for $v_{\text {DS }}$ voltage waveforms for devices with (a) lossless and (b) lossy $C_{\mathrm{OSs}}$ charging/discharging processes. (d) Areas below $v_{\mathrm{DS}}$ waveform for charging $\left(S_{1}\right)$ and discharging $\left(S_{2}\right)$ processes translates to (d) $C_{\mathrm{OSs}}$ charging/discharging energies as well as the dissipated energy $E_{\text {DIss. }}$.

well as $E_{\text {DIss. }}$ The circuit has a high step-up which enables to probe $C_{\mathrm{OSS}}$ and $E_{\mathrm{DISS}}$ at very large voltages [13]. It is important to note that the resonant nature of the circuit guarantees lossless soft switching at $t=t_{0}$. In addition, the losses related to the internal gate resistance of transistor and the sink resistance of the gate driver are negligible. The governing equation of the circuit shown in Fig. 1(a) is

$$
L C\left(v_{\mathrm{DS}}\right) \frac{\mathrm{d} v_{\mathrm{DS}}}{\mathrm{d} t}=L I_{0}-\int_{t_{0}}^{t} v_{\mathrm{DS}}(t) \mathrm{d} t
$$

where $C=C_{\mathrm{oss}}+C_{\mathrm{par}}$ is the total nonlinear voltage-dependent capacitance at the output node. When $v_{\mathrm{DS}}$ peaks at $t=0$, the inductor has zero current, so the inductor flux $\left(\varphi=L I_{0}\right)$ can be calculated by integration of its voltage. Since $v_{\mathrm{DD}} \ll v_{\mathrm{DS}}$ :

$$
L I_{0}=\int_{t_{0}}^{0} v_{\mathrm{DS}}(t) \mathrm{d} t .
$$

Replacing (2) into (1), yields

$$
C\left(v_{\mathrm{DS}}\right)=\frac{\int_{t_{0}}^{0} v_{\mathrm{DS}}(t) \mathrm{d} t-\int_{t_{0}}^{t} v_{\mathrm{DS}}(t) \mathrm{d} t}{L \frac{\mathrm{d} v_{\mathrm{DS}}}{\mathrm{d} t}} .
$$

Equation (3) presents the expression of the capacitance $C$ exclusively as a function of $v_{\mathrm{DS}}$. The inductance value of $L$ in the denominator of (3) should be properly measured to accurately extract the large-signal $C_{\text {Oss. }}$. It should be noted that $v_{\mathrm{DS}}$ starts from 0 at $t=t_{0}$, increases to the maximum value of $V_{\max }$ at $t=0$, and then again, decreases to 0 . This results in a double-sweep large-signal measurement of output capacitance for each pulse. In the case of devices without $C_{\text {oss }}$ hysteresis, the measured $v_{\mathrm{DS}}$ has a symmetric waveform (Fig. 1(b)), while devices with $C_{\text {Oss }}$ hysteresis present a non-symmetric waveform (Fig. 1(c)).

Equation (3) can be used to calculate the energy transferred to (or recovered from) $C_{\text {OSs }}$ during a time interval $T$

$$
E_{T}=\int_{T} v_{\mathrm{DS}} C\left(v_{\mathrm{DS}}\right) \mathrm{d} v_{\mathrm{DS}}
$$

After replacing (3) into (4), the $C_{\text {oss }}$ charging $\left(T=\left[t_{0}, 0\right]\right)$ and discharging $\left(T=\left[0, t_{1}\right]\right)$ energies can be extracted as

$$
E_{\mathrm{OSS}}^{\mathrm{ch}}=\frac{1}{2 L}\left(\int_{t_{0}}^{0} v_{\mathrm{DS}}(t) \mathrm{d} t\right)^{2}
$$

and

$$
E_{\mathrm{OSS}}^{\text {disch }}=\frac{1}{2 L}\left(\int_{0}^{t_{1}} v_{\mathrm{DS}}(t) \mathrm{d} t\right)^{2}
$$

respectively. The difference between (5) and (6) gives the energy dissipated in $C_{\text {oss }}$

$$
E_{\text {DISS }}=\frac{1}{2 L}\left(\left(\int_{t_{0}}^{0} v_{\text {DS }}(t) \mathrm{d} t\right)^{2}-\left(\int_{0}^{t_{1}} v_{\text {DS }}(t) \mathrm{d} t\right)^{2}\right)
$$

Figs. 1(d) and (e) depict the correspondence of the $C_{\text {OSS }}$ charging/discharging/dissipated energies to the areas below the $v_{\mathrm{DS}}$ curve. This makes the method numerically convenient since it just relies on the integration of a single voltage waveform, which can be accurately measured even at ultra-fast charging/discharging cycles, even below 10 ns. It should be noted that the use of a high- $Q$ inductor $L$ leads to negligible deformation of $v_{\mathrm{DS}}$ waveform, even below the measurement accuracy of typical oscilloscopes.

\section{EXPERIMENTS}

We demonstrate the applicability of the proposed method for large-signal $C_{\mathrm{OSS}}$ measurement as well as to extract $E_{\mathrm{DISS}}$ for different transistors (listed on Table I). Fig. 2a shows the experimental set-up with different test boards compatible with different transistor packages. The captured $v_{\mathrm{DS}}$ and $v_{\mathrm{GS}}$ waveforms verify the soft switching in the circuit since the transistor turns OFF $\left(v_{\mathrm{GS}}=0\right)$ before building-up the $v_{\mathrm{DS}}$ voltage. A test board with 1-mH inductor is shown in Fig. $2 \mathrm{~b}$. The impedance of the employed inductor was measured using a Keysight E4990A impedance analyzer, as shown in Fig. 2c.

\begin{tabular}{|c|c|c|c|c|}
\hline \multirow{2}{*}{ No. } & \multirow{2}{*}{ Technology } & \multicolumn{2}{|c|}{ Voltage/Current Ratings } & \multirow{2}{*}{$\begin{array}{c}\text { Reported } E_{\text {Oss }} \text { at } \\
400 \mathrm{~V}(\mu \mathrm{J})\end{array}$} \\
\hline & & Voltage (V) & Current (A) & \\
\hline M1 & $\mathrm{SiC}$ & 650 & 93 & 22.0 \\
\hline M2 & Si SJ & 650 & 60 & 17.5 \\
\hline M3 & $\mathrm{SiC}$ & 900 & 36 & 8.5 \\
\hline M4 & $\mathrm{GaN}$ & 650 & 65 & 15.5 \\
\hline M5 & $\mathrm{GaN}$ & 650 & 65 & 7.0 \\
\hline M6 & $\mathrm{GaN}$ & 600 & 30 & 3.0 \\
\hline
\end{tabular}
Based on this measurement, we extracted and de-embedded the parasitic capacitance of the inductor (inset of Fig. 2c). The results for $\mathrm{M} 1(\mathrm{SiC})$ and $\mathrm{M} 2(\mathrm{Si} \mathrm{SJ})$ using $1-\mathrm{mH}$ inductor, shown in Figs. 3 (a) and (c), respectively, illustrate the shape of

TABLE I

SPECIFICATIONS OF THE EVALUATED TRANSISTORS
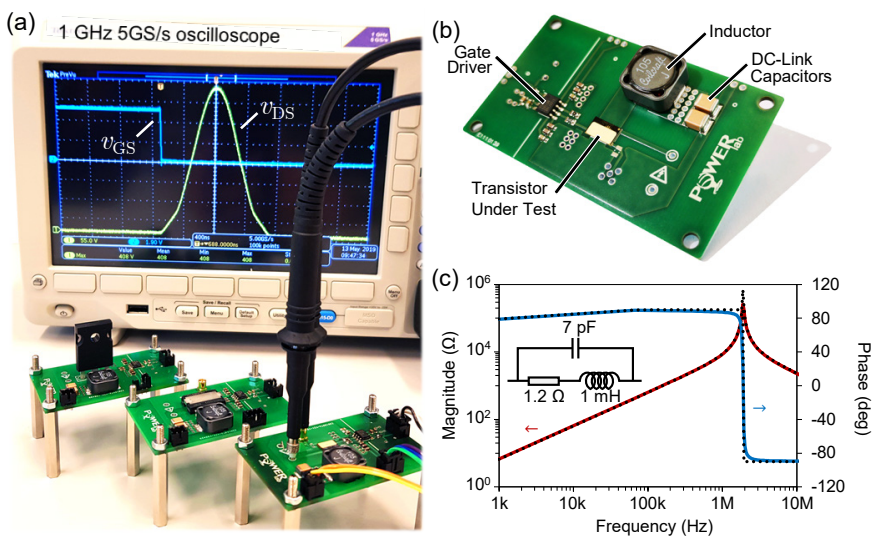

Fig. 2. Photographs of (a) the experimental set-up and (b) a test board. (c) Measured (solid lines) and modeled (dotted lines) impedance of a 1-mH inductor. The inset shows the equivalent circuit model of the inductor. 

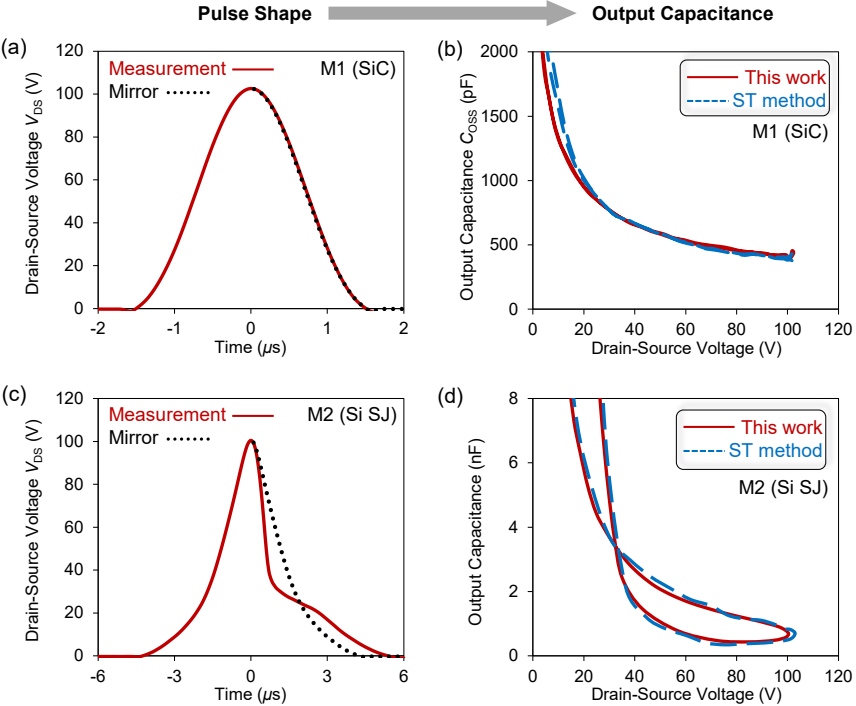

Fig. 3. (a) Measured $v_{\mathrm{DS}}$ (solid line) and mirrored waveform (dotted line) for M1. (b) Extracted $C_{\mathrm{Oss}}$ for M1 using the proposed method (solid line) and by ST method (dashed line). (c) Measured $v_{\text {DS }}$ (solid line) and mirrored waveform (dotted line) for M2 shows a non-symmetrical charging/discharging process. (d) Extracted $C_{\text {Oss }}$ for M1 using the proposed method (solid line) and by ST method (dashed line).

the $v_{\mathrm{DS}}$ pulse for these two transistors, which translates into large-signal $C_{\text {oss }}$ (Figs. 3 (b) and (d), respectively). The obtained large-signal $C_{\text {oss }}$ were verified with ST method performed at $300 \mathrm{kHz}$ (dashed lines in Figs. 3 (b) and (d)). It should be noted that although the measurements were done at $V_{\max }=100 \mathrm{~V}$, the supply voltage $v_{\mathrm{DD}}$ was very small, e.g. $v_{\mathrm{DD}}=$ $100 \mathrm{mV}$ in Fig. 3(a).

The large values of inductor $L$ result in relatively small resonance frequencies, e.g. $300 \mathrm{kHz}$ for $\mathrm{M} 1 \mathrm{(SiC}$ ) in Fig. 3(a), however, much higher frequencies and $\mathrm{d} v / \mathrm{d} t$ values can be obtained with smaller inductors (Fig. 4(a)). Fig. 4(b) shows measurement results for M2 (Si SJ) with a 500-nH air-core inductor. By changing the small voltage $v_{\mathrm{DD}}$, the peak voltage $V_{\max }$ was tuned at $250 \mathrm{~V}, 450 \mathrm{~V}$, and $650 \mathrm{~V}$. Fig. 4(c) shows the extracted large-signal $C_{\text {oss, }}$, presenting considerable hysteresis loops. The reported small-signal $C_{\text {oss }}$ from the device datasheet (discrete points in Fig. 4(c)) closely match the extracted largesignal $C_{\text {OSs }}$ in the discharge cycle.

The method is capable of being performed at high voltage levels. Fig. 4(d) shows the measured $v_{\mathrm{DS}}$ waveforms with $L=$ $500 \mathrm{nH}$ at three different voltage $200 \mathrm{~V}, 600 \mathrm{~V}$, and $1000 \mathrm{~V}$, and the corresponding large-signal capacitances are shown in Fig. 4(e). For the $1000 \mathrm{~V}$ measurement, a resonance frequency of $40 \mathrm{MHz}$ and $\mathrm{d} v / \mathrm{d} t$ of $120 \mathrm{~V} / \mathrm{ns}$ show the potential of the method in operating at very high frequencies and amplitudes, at the same time, without the risk of thermal runaway. To put these numbers in perspective, with the same measurement specifications for a device with an output capacitance of $\sim 100$ $\mathrm{pF}$, the amplifier in the ST method would require to provide $\sim 3$ $\mathrm{kVA}$ of reactive power at $40 \mathrm{MHz}$.

The use of GaN-on-Si HEMTs in high-frequency converters is rapidly growing, due to their outstanding features such as low $R_{\mathrm{ON}}$ and small gate charge. Unexpected losses in soft-switched GaN-based power converters initiated studies on $C_{\text {Oss }}$ losses in these devices [2]. Figs. 5(a) and (b) show the measured voltage waveforms over a cascode GaN transistor (M4), at low and high
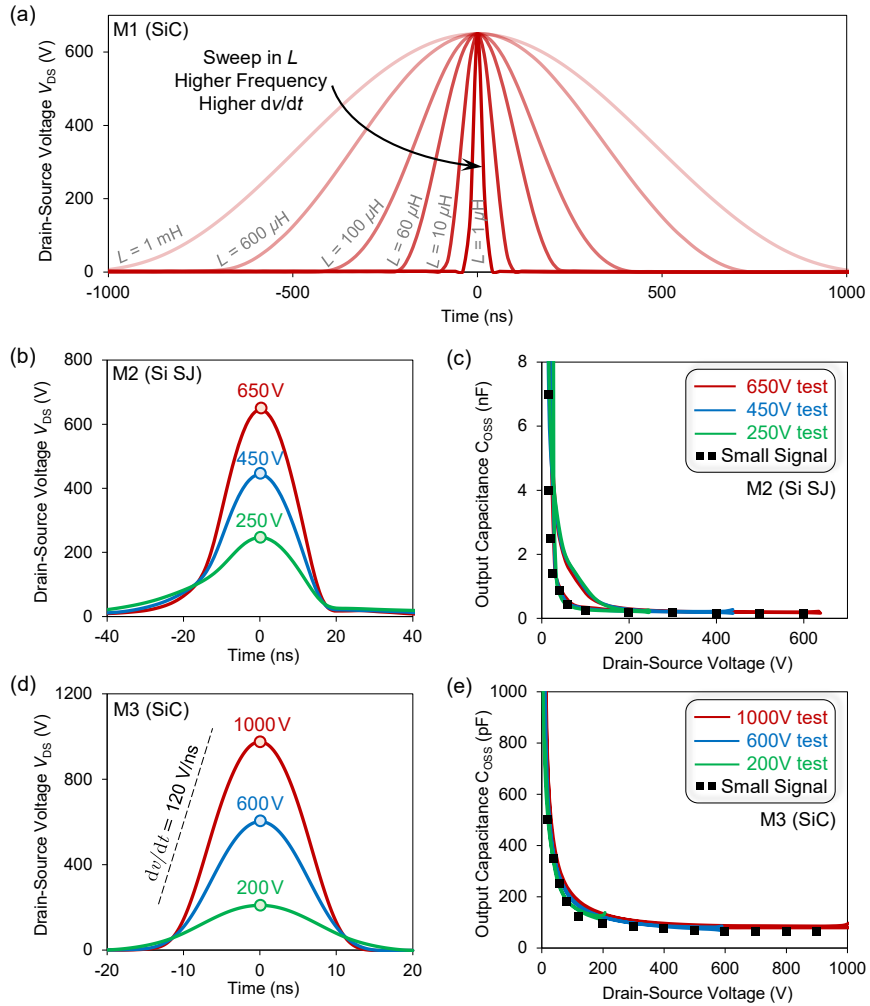

Fig. 4. (a) Measured vDS for M1 (SiC) using different values of L showing the possibility of capturing large-signal $C_{\mathrm{OSS}}$ and $E_{\mathrm{DISS}}$ at very frequencies. (b) Measured $v_{\mathrm{DS}}$ for M2 (Si SJ) at $V_{\max }=250 \mathrm{~V}, 450 \mathrm{~V}$, and $650 \mathrm{~V}$, and (c) the corresponding large-signal $C_{\text {OSs. }}$ (d) Measured $v_{\mathrm{DS}}$ for $\mathrm{M} 3$ (SiC) at $V_{\max }=200$ $\mathrm{V}, 600 \mathrm{~V}$, and $1000 \mathrm{~V}$, and (e) the corresponding large-signal $C_{\text {oss. }}$. Discrete points show small signal $C_{\mathrm{OsS}}$ reported in datasheets.
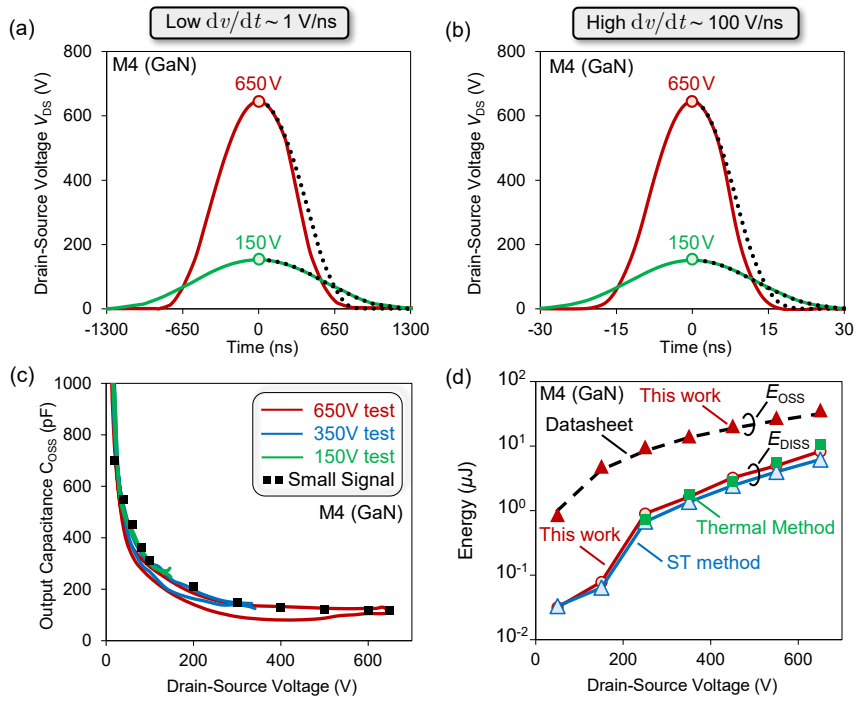

Fig. 5. Capturing large-signal $C_{\mathrm{OSS}}$ and $E_{\mathrm{DISS}}$ at low and high $\mathrm{d} v / \mathrm{d} t$ values for M4 (GaN). (a) Low and (b) high $\mathrm{d} v / \mathrm{d} t$ measurements with $L=1 \mathrm{mH}$ and $L=$ $500 \mathrm{nH}$ inductors, respectively. The mirrors (dotted lines) show symmetry at low voltage tests $(150 \mathrm{~V})$ and non-symmetry at high voltage tests $(650 \mathrm{~V})$. Large signal $C_{\mathrm{Oss}}$ and $E_{\mathrm{DISs}}$ are not functions of $\mathrm{d} v / \mathrm{d} t$ in this $\mathrm{GaN}$ transistor. (c) Extracted large signal $C_{\mathrm{OSS}}$ for $V_{\max }=150 \mathrm{~V}, 350 \mathrm{~V}$, and $650 \mathrm{~V}$. (d) Extracted losses from the proposed technique, validated with ST and thermal method.

$\mathrm{d} v / \mathrm{d} t$ values, respectively. At $150-\mathrm{V}$ tests, the generated $v_{\mathrm{DS}}$ waveforms are almost symmetrical for both low and high $\mathrm{d} v / \mathrm{d} t$ tests. At a higher voltage $V_{\max }=650 \mathrm{~V}$, however, there is a significant asymmetry in both waveforms. Fig. 5(c) presents the extracted large-signal $C_{\mathrm{OSs}}$ for $V_{\max }=150 \mathrm{~V}, 350 \mathrm{~V}$, and $650 \mathrm{~V}$, showing that the hysteresis loop becomes drastically larger at 

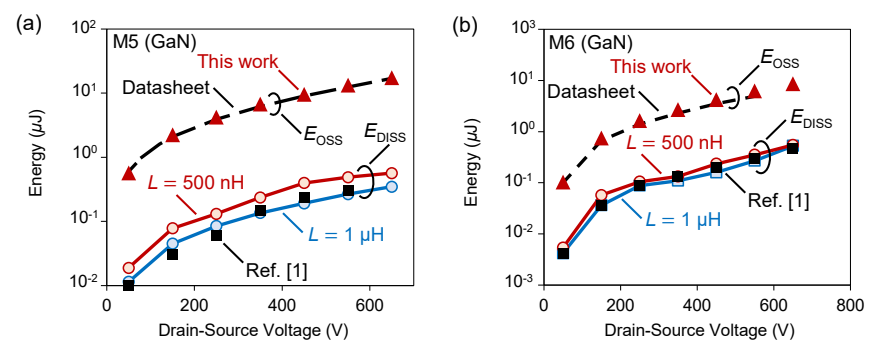

Fig. 6. Extracting $E_{\mathrm{OSS}}$ and $C_{\mathrm{OSs}}$ losses for enhancement-mode GaN transistors (a) M5 and (b) M6, for $L=500 \mathrm{nH}$ and $L=1 \mu \mathrm{H}$. The extracted $E_{\text {OSS }}$ values $(\Delta)$ (equal for both cases $L=500 \mathrm{nH}$ and $1 \mu \mathrm{H}$ ) are in agreement with reported data in datasheets (dashed lines) and the obtained $E_{\text {DIss }}$ for $L=1 \mu \mathrm{H}(f$ $\sim 10 \mathrm{MHz}$ ) shows a good agreement with reported data in [1] ( $\square$ ).

higher voltages. This indicates a fundamentally different loss mechanism than that in SJ devices, since the device becomes significantly lossy at high voltages. The extracted $E_{\mathrm{OSS}}$ and $C_{\mathrm{OSS}}$ losses at different voltage levels are shown in Fig. 5(d). The significant increase in $C_{\mathrm{OSS}}$ losses appearing for $v_{\mathrm{DS}}>250 \mathrm{~V}$, is in agreement with the previously measured $C_{\text {oss }}$ losses for cascode GaN devices [1]. The extracted $E_{\text {DISs }}$ based on the proposed method, were verified with thermal method and ST measurements performed at a wide range of different frequencies from $10 \mathrm{kHz}$ to $300 \mathrm{kHz}$. The agreement between $E_{\text {Oss }}$ reported in datasheet (dashed line in Fig. 5(d)) and the extracted $E_{\text {Oss }}$ from (5) ( $\Delta$ in Fig. 5(d)) is another indication of the accuracy of the proposed method. At the highest test voltage of $650-\mathrm{V}, 26 \%$ of $E_{\mathrm{OSS}}$ is dissipated during charging and discharging processes. Although $\mathrm{d} v / \mathrm{d} t$-dependent losses have been observed in some of the advanced GaN HEMTs [1], [2], as it can be seen in Figs. 5(a) and (b), the proposed method did not show any significant difference for $\mathrm{GaN}$ device $\mathrm{M} 4$, which opens opportunities for further investigation of these effects on different devices.

Figs. 6(a) and (b), show $E_{\text {OSs }}$ and single-cycle charging/discharging losses of enhancement-mode $\mathrm{GaN}$ transistors M5 and M6, respectively. The extracted $E_{\mathrm{OSS}}(\Delta)$ for both devices matches well with data reported in their datasheets

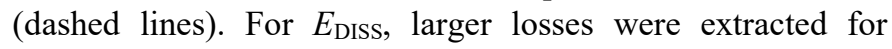
smaller inductor $L=500 \mathrm{nH}$, corresponding to higher $\mathrm{d} v / \mathrm{d} t$. The obtained losses for M5 and M6 are considerably lower than losses detected for M4, e.g. $3.5 \%$ of nominal $E_{\mathrm{OSS}}$ at $650 \mathrm{~V}$ for M5. The obtained results, for $L=1 \mu \mathrm{H}$ (leading to a resonance frequency of $\sim 10 \mathrm{MHz}$ ) are in agreement with previously reported $C_{\mathrm{Oss}}$ losses for M5 and M6 at the same frequency [1].

\section{CONCLUSION}

In this work we demonstrated a new measurement technique to evaluate the large-signal $C_{\mathrm{OSS}}$ and $C_{\mathrm{OSs}}$ losses of transistors. The method has several advantages

1) Using a low-voltage DC source, high voltage swings ( $>$ $1000 \mathrm{~V})$, high frequencies ( $>40 \mathrm{MHz}$ ), and high values of $\mathrm{d} v / \mathrm{d} t(>120 \mathrm{~V} / \mathrm{ns})$ can obtained.

2) The method can be performed in single-pulse mode which eliminates thermal issues like thermal runaway or increasing leakage at high temperatures, even at very high voltage swings and high values of $\mathrm{d} v / \mathrm{d} t$.

3) Unlike ST, where the circuit is not representative of the operating conditions of a device in a switching circuit [2], the nature of the proposed circuit has similarities to the concept of resonant converters.

4) The simplicity of the circuit, relying on just two main elements (inductor and DUT), minimizes the parasitic effects in waveforms. For instance, any kind of voltage ringing over DUT in more complicated circuits leads to partial $C_{\text {OSS }}$ charging/discharging that causes additional losses. These ringings are more dominant in high $\mathrm{d} v / \mathrm{d} t$ and can potentially affect the accuracy of measurement. For the proposed method, however, there is always one smooth charging and discharging curve, without any ringing.

Evaluation of a cascode $\mathrm{GaN}$ transistor with the proposed method, showed $\mathrm{d} v / \mathrm{d} t$-independent $C_{\mathrm{OSs}}$ loss that was drastically increased after $250 \mathrm{~V}$, reaching $26 \%$ of $E_{\text {oss }}$ at the maximum rating voltage of $650 \mathrm{~V}$ (M4). Considerably smaller $C_{\text {Oss }}$ losses was measured for two enhancement-mode GaN transistors (M5 and M6). The

simplicity and consistency of the proposed method enables the study of large-signal $C_{\mathrm{OSs}}$ and $C_{\mathrm{OSS}}$ losses of transistors, which can significantly influence the performance of power converters, especially those operating at high switching frequencies.

\section{REFERENCES}

[1] G. Zulauf, S. Park, W. Liang, K. Surakitbovorn, and J. Rivas-Davila, " $C_{\text {oss }}$ losses in $600 \mathrm{~V} \mathrm{GaN}$ power semiconductors in soft-switched, highand very-high-frequency power converters," IEEE Trans. Power Electron., vol. 33, no. 12, pp. 10748-10763, Dec. 2018.

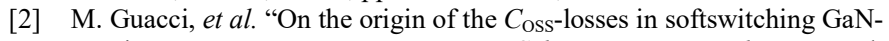
on-Si power HEMTs," IEEE J. Emerg. Sel. Topics Power Electron., vol. 7, no. 2, pp. 679-694, Jun. 2019.

[3] J. Roig and F. Bauwens, "Origin of anomalous $C_{\text {oss }}$ hysteresis in resonant converters with superjunction FETs," IEEE Trans. Electron Devices, vol. 62, no. 9, pp. 3092-3094, Sep. 2015.

[4] G. D. Zulauf, J. Roig-Guitart, J. D. Plummer, and J. M. Rivas-Davila, " $C_{\mathrm{OSS}}$ measurements for superjunction MOSFETs: Limitations and opportunities," IEEE Trans. Electron Devices, vol. 66, no. 1, pp. 578584, Jan. 2019.

[5] G. Zulauf, Z. Tong, J. D. Plummer, and J. M. Rivas-Davila, "Active power device selection in high- and very-high-frequency power converters," IEEE Trans. Power Electron., vol. 34, no. 7, pp. 6818-6833, Jul. 2019.

[6] D. Rothmund, D. Bortis, and J. W. Kolar, "Accurate transient calorimetric measurement of soft-switching losses of $10 \mathrm{kV} \mathrm{SiC} \mathrm{MOSFETs} \mathrm{and}$ diodes," IEEE Trans. Power Electron., vol. 33, no. 6, pp. 5240-5250, Jun. 2018.

[7] M. Kasper, R. M. Burkart, G. Deboy, and J. W. Kolar, "ZVS of power MOSFETs revisited," IEEE Trans. Power Electron., vol. 31, no. 12, pp. 8063-8067, Dec. 2016.

[8] J. Fedison and M. Harrison, " $C_{\text {oss }}$ hysteresis in advanced superjunction MOSFETs," Appl. Power Electron. Conf. Expo., 2016, pp. 247-252.

[9] J. Fedison, M. Fornage, M. Harrison, and D. Zimmanck, " $\mathrm{C}_{\text {oss }}$ related energy loss in power MOSFETs used in zero-voltage-switched applications," in Proc. 29th Annu. IEEE Appl. Power Electron. Conf. Expo., 2014, pp. 150-156.

[10] Z. Tong, G. Zulauf, J. Xu, J. D. Plummer, and J. Rivas-Davila, "Output capacitance loss characterization of silicon carbide schottky diodes," IEEE Trans. Power Electron., IEEE J. Emerg. Sel. Topics Power Electron., vol. 7, no. 2, pp. 856-878, Jun. 2019.

[11] S. Ben-Yaakov, "Some observations on loss and hysteresis of ferroelectric based ceramic capacitors," IEEE Trans. Power Electron., vol. 33, no. 11, pp. 9127-9129, Nov. 2018.

[12] J. F. Scott, "Ferroelectrics go bananas," J. Phys. Condens. Matter, vol. 20, no. 2, p. 021001 , Dec. 2007.

[13] M. S. Nikoo, A. Jafari, and E. Matioli, "GaN transistors for miniaturized pulsed-power sources," IEEE Trans. Plasma Sci., early access, 2019. DOI: 10.1109/TPS.2019.2917657. 